American Journal of Biochemistry and Biotechnology 2 (2): 73-79, 2006

ISSN 1553-3468

(c) 2005 Science Publications

\title{
Static and Dynamic Mechanical Behavior of Hydroxyapatite-Polyacrylic Acid Composites Under Simulated Body Fluid
}

\author{
Kalpana S. Katti, Phanikumar Turlapati, Devendra Verma, \\ Rahul Bhowmik, Praveen K. Gujjula, and Dinesh R. Katti \\ Department of Civil Engineering, North Dakota State University, Fargo, ND 58105
}

\begin{abstract}
In this work, we have investigated mechanical response of hydroxyapatite/polyacrylic composites under dry, wet and simulated body fluid conditions. Hydroxyapatite (HAP) is mineralized under two conditions; one, in presence of polyacrylic acid (in situ HAP), second, in absence of polyacrylic acid (ex situ HAP). Further, in situ and ex situ HAP are mixed with polyacrylic acid to make HAP/PAAc composites. Interfacial interactions between PAAc and HAP have been studied using photoacoustic Fourier transform infrared spectroscopy (PAS-FTIR). The mechanical response of the composites under wet condition is studied by soaking composite samples in simulated body fluid (SBF). Under wet conditions, SBF and water weaken the HAP-HAP interface significantly. PAS-FTIR data suggests that PAAc attaches to HAP through the dissociated carboxylate groups. The water and SBF soaked samples showed creep-like behavior and exhibit large residual strain after unloading. Loading under different strain rates has significant effect on mechanical properties of these composites. Both in situ and ex situ 70:30 composites exhibit highest elastic modulus at strain rate of $0.01 \mathrm{sec}-1$. XRD study indicates formation of $\mathrm{Ca}_{2} \mathrm{P}_{2} \mathrm{O}_{7}$ phase in ex situ composite after soaking in SBF and water for 3 hours, whereas in situ composites showed presence of only hydroxyapatite phase after soaking in SBF and water for same duration of time.
\end{abstract}

Key words: biomaterials, hydroxyapatite, polyacrylic acid, simulated body fluid

\section{INTRODUCTION}

Biological composites are ideal model systems because of their optimized properties in combination with hierarchy and adaptability ${ }^{[1-4]}$. In biological composites, the mineralization occurs in close association with organic polymers ${ }^{[5,6]}$. The organic phase regulates the particle size, shape, crystal orientation, polymorphic structure, defect texture, and particle assembly of the mineral ${ }^{[7-9]}$. Especially significant is the fact that the processes occur at physiological conditions. Synthetic materials based on biological materials have led to design of a new class of novel materials called biomimetic materials. Seemingly, it would be difficult to mimic complex cellular processes; however, the materials chemistry aspects of biomineralization are being studied to develop biomimetic composites ${ }^{[10-13]}$. The results of using this technology in laminated biocomposites mimicking seashells have shown great promise ${ }^{[14]}$. The essence of this process lies in mimicking biological mineralization in which a pre-organized organic phase provides a template for inorganic crystals to nucleate and grow from supersaturated solution. A molecular control of crystallography and morphology of the inorganic phase would allow material design with tailored properties. Several studies have been conducted to study the effect of various organic additives on the mineralization of HAP ${ }^{[15-17]}$. The structural control on morphology, size, and orientation of the inorganic crystallites is the main role of the organic additive. Current research efforts have thus focused on composites of synthetic polymers and HAP formed using in situ mineralization techniques. A few polymeric additives such as poly(acrylic acid) (PAAc) and gelatin have been investigated as suitable organic additives ${ }^{[18,19]}$. Specifically, polyacrylic acid has been found to accelerate the nucleation of HAP from calcium and phosphate solutions. The ionizable carboxylate functionalities of PAAc provide high affinity to calcium ions from HAP [16, 20-23]. Our recent work using molecular dynamics simulation has elucidated the presence of chelation and hydrogen bonds at mineralpolymer interfaces in in situ composites ${ }^{[24]}$. The applicability of in situ HAP composites as scaffolds for bone tissue engineering has been investigated ${ }^{[22,23]}$. The presence of PAAc in biomimetic HAP significantly alters its surface properties. Mechanical properties of such composites are still inadequate for their potential use for bone replacement. The specific knowledge of the fundamental mechanism of the association of organic functionalities with HAP during HAP nucleation and growth is lacking.

In this work, composites of hydroxyapatite and polyacrylic acid are biomimetically synthesized by

Corresponding Author: Kalpana S. Katti, Department of Civil Engineering, North Dakota State University, Fargo ND 58105. Tel.: 1-701-231-9504; Fax: 1-701-231-6185 
mineralizing hydroxyapatite in the presence of polyacrylic acid (in situ HAP). Hydroxyapatite was also synthesized in absence of polyacrylic acid (ex situ HAP). Further, composites of in situ and ex situ HAP with PAAc were made by mixing. The interfacial interaction between PAAc and HAP has been investigated using photoacoustic Fourier transform infrared (PA-FTIR) spectroscopy. Since, these composites are investigated for bone implant application and expected to function under aqueous body conditions, the mechanical response after soaking in simulated body fluid (SBF) and water has also been studied. The rate dependent properties of the composites are analyzed at varying strain rates.

\section{MATERIALS AND METHODS}

\section{Materials}

$\mathrm{Na}_{2} \mathrm{HPO}_{4}, \mathrm{NaHCO}_{3}$ and TRIS were obtained from J.T. Baker, $\mathrm{KCl}, \mathrm{MgCl}_{2} .8 \mathrm{H}_{2} \mathrm{O}, \mathrm{Na} 2 \mathrm{SO} 4$ and $\mathrm{CaCl}_{2}$ were obtained from EM Science and $\mathrm{NaCl}$ and $\mathrm{K}_{2} \mathrm{HPO} .3 \mathrm{H}_{2} \mathrm{O}$ were obtained from Mallinckrodt. Polyacrylic acid (Mn $=450,000 \mathrm{~g} / \mathrm{mol}$ ) was obtained from Polysciences Inc.

\section{Ex situ and in situ HAP Preparation}

Ex situ HAP is prepared by mixing $\mathrm{Na}_{2} \mathrm{HPO}_{4}$ and $\mathrm{CaCl} 2$ solutions in stoichiometric ratio. The resulting solution was kept undisturbed for $24 \mathrm{~h}$ and then HAP precipitate was separated by centrifuging. For in situ HAP preparation, $0.5 \mathrm{gm}$ of PAAc was dissolved in 1 liter Na2HPO4 solution $(7.2 \mathrm{mM})$ before addition of $\mathrm{CaCl} 2$ solution $(12 \mathrm{mM})$. The $\mathrm{pH}$ was maintained at 7.4 using $1 \mathrm{~N} \mathrm{NaOH}$. The appearance of turbidity signals the formation of amorphous calcium phosphate. A drop in $\mathrm{pH}$ signals the amorphous to crystalline transformation.

\section{Composite fabrication}

For composite fabrication, the $\mathrm{HAP} /$ polymer ratio is maintained at 70/30 and 60/40 wt $\%$.

The mineralized precipitates and PAAc in the above ratios are mixed using a mortar and pestle. Further, thoroughly mixed powders were compression molded in cylindrical shape with 0.5 " diameter and 1" height at ambient temperature and $80 \mathrm{MPa}$.

\section{Experimental analysis}

X-ray powder diffraction studies of in situ and ex situ HAP and their composites were carried out using a Philips diffractometer using $\mathrm{Cu} K \alpha$ radiation $(\lambda=$ $1.5405 \AA$ ). Compression tests of solid composite samples were done at a loading rate of $50 \mathrm{~N} /$ using SATEC $^{\text {TM }}$ materials testing servo mechanical test frame. Elastic modulus has been determined from the slope of initial linear portion of stress-strain curves. PA-FTIR spectroscopy study was done using Thermo Electron, Nexus 870 spectrometer equipped with MTEC Model 300 photoacoustic accessory. Linear photoacoustic spectra (500 scans) of all samples were collected in the range of $4000-400 \mathrm{~cm}^{-1}$ at a mirror velocity of $0.15 \mathrm{~cm} / \mathrm{s}$.

\section{Modeling Approach}

In modeling approach, the conformation of ionized and unionized PAAc was analyzed for the mineralization of HAP. The ionized PAAc consists of with ionized carboxylate groups (COO-). Here molecular dynamics simulations were performed using CVFF (Consistent Valence Forcefield) potential function. The CVFF force is commonly used for molecular dynamics simulations for a variety of polymeric and mineral materials ${ }^{[24-36]}$. The details for the derivation of parameters in CVFF potential function for HAP is described in our previous study ${ }^{[24,32]}$.

\section{RESULTS AND DISCUSSION}

Figure 1 shows linear PAS-FTIR spectra of in situ HAP/PAAc composite, ex situ HAP/PAAc composite and PAAc obtained at mirror velocity $0.15 \mathrm{~cm} / \mathrm{s}$. The bands at $550 \mathrm{~cm}^{-1}$ and $1050 \mathrm{~cm}^{-1}$ originate from bending and stretching vibration mode of phosphate of HAP respectively. The bands at $1454 \mathrm{~cm}^{-1}$ and $1412 \mathrm{~cm}^{-1}$ have been assigned to scissoring of $-\mathrm{CH}-$ and bending of $-\mathrm{CH}-\mathrm{CO}-$ of PAAc. The broad band at around 3300 $\mathrm{cm}^{-1}$ is due to stretching of $\mathrm{OH}$ of carboxylate groups and adsorbed water. The band at $1702 \mathrm{~cm}^{-1}$ is assigned to stretching from carbonyl of $-\mathrm{COOH}$. Another band is observed at $1555 \mathrm{~cm}^{-1}$ in both the in situ and ex situ HAP/PAAc composites. This band has been assigned to asymmetric carbonyl stretching of dissociated carboxylate groups. This band is also present in PAAc but its intensity is extremely low. This band appears when $-\mathrm{COOH}$ moieties dissociate to $-\mathrm{COO}^{-}$. The dissociated carboxylate groups act as favorable sites for nucleation of hydroxyapatite. Dissociated carboxylate groups form complexes with $\mathrm{Ca}^{2+}$ in the solution and facilitate growth of hydroxyapatite crystals.

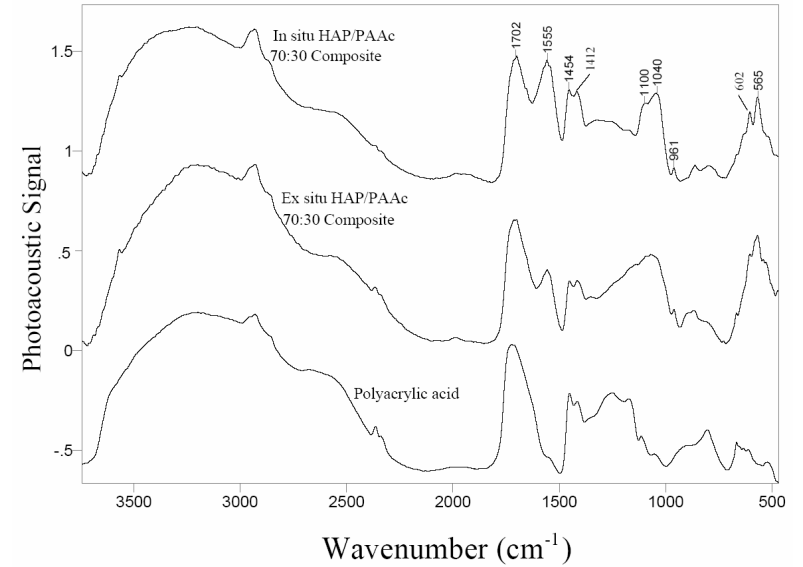

Fig. 1: PAS-FTIR spectra of in situ HAP/PAAc composite, ex situ HAP/PAAc composite and PAAc. 
In order to understand the attachment of different ions on ionized PAAc, it is necessary to identify the structure of PAAc in ionized and unionized states. In our study, molecular mechanics simulations were performed on PAAc, which includes minimization and MD simulations. We have used ten different conformation of ionized and unionized PAAc. Each of the structure was first minimized then MD simulations were performed at $300 \mathrm{~K}$ for $50 \mathrm{ps}$. Further, the temperature of the structure is raised to $500 \mathrm{~K}$ and back to $300 \mathrm{~K}$ in $50 \mathrm{~K}$ steps. At each temperature, MD simulations were performed using NVT ensemble for time period of 50 ps. Finally, PAAc was minimized again. This is performed to obtain conformations of PAAc at global minimum of energy. Here the minimization was initiated with steepest descent method followed by conjugate gradient with convergence from $1000 \mathrm{kcal} \mathrm{mol}^{-1} \AA^{-1}$ to $0.001 \mathrm{kcal} \mathrm{mol}^{-}$ ${ }^{1} \AA^{-1}$. The energy of each structure at the start and at the end is shown in Table 1.

Table 1(a): Initial and final energy of ten different conformation of unionized PAAc

\begin{tabular}{c|c|c}
\hline $\begin{array}{c}\text { Unionized } \\
\text { PAAc }\end{array}$ & $\begin{array}{c}\text { Initial Energy } \\
\text { (kcallmol) }\end{array}$ & $\begin{array}{c}\text { Final Energy } \\
(\mathrm{kcal} / \mathrm{mol})\end{array}$ \\
\hline \hline 1 & 253.87 & 196.90 \\
2 & 251.53 & 191.48 \\
3 & 228.60 & 199.62 \\
4 & 206.67 & 198.90 \\
5 & 221.93 & 201.89 \\
6 & 214.67 & 214.05 \\
7 & 212.76 & 196.48 \\
8 & 214.48 & 203.83 \\
9 & 238.12 & 193.52 \\
10 & 234.61 & 202.43 \\
\hline
\end{tabular}

Table 1(b): Initial and final energy of ten different conformation of ionized PAAc

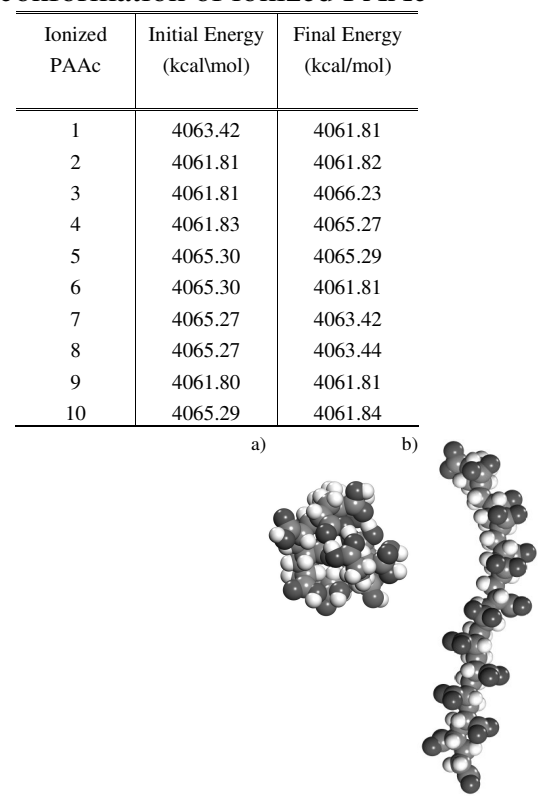

Fig. 2: Structure of PAAc $(\mathrm{O}=$ dark grey, $\mathrm{C}=$ light grey, and $\mathrm{H}=$ white) a) unionized $\mathrm{b}$ ) ionized
Each of the final structures of unionized PAAc exhibit spherical shape whereas the structures of ionized PAAc exhibit linear shape (Figure 2). The open structure of ionized PAAc (linear shape) results from a repulsive behavior of ionized carboxylate groups; due to which more ionized carboxylate groups tend to interact with ions in the solution while synthesizing in situ HAP. Here, first $\mathrm{Ca}^{+2}$ ions from HAP interact with ionized carboxylate groups of PAAc. The structure of PAAc showing lowest energy (both ionized and unionized states) was used for further simulations with HAP. Here we have used structures 2 and 6 for unionized and ionized states of PAAc. These structures have lower energies as compared to other structures (Table 1). Our simulations have also indicated that ionized carboxylate groups interact with calcium ions and form chelating structures $^{[27]}$.

From the PAS-FTIR spectra it is observed that the dissociated carboxylate groups also show a band at around $1340 \mathrm{~cm}^{-1}$, which has been assigned to symmetric stretching of carbonyl. Presence this band in ex situ HAP/PAAc composite suggests that at the interface, carboxylate groups dissociate and chelate with $\mathrm{Ca}$ atoms present on surface of ex situ HAP particles which is also observed in modeling studies. Intensity of this band is higher in in situ HAP/PAAc composite than ex situ HAP/PAAc composite. This is due to the fact that not only do the carboxylate groups of in situ HAP chelate with $\mathrm{Ca}$ atoms of hydroxyapatite as indicated by simulations and PA-FTIR spectrum but also that PAAc of composite chelates with $\mathrm{Ca}$ atoms present on surface of in situ HAP. The carboxylate groups present on surface of in situ HAP could also hydrogen bond with carboxylate groups of in situ HAP/PAAc composite. Figure 3 shows stress/strain plots of in situ and ex situ composite samples. Both samples show a characteristic two-stage behavior. The elastic modulus of in situ HAP/PAAc composite is higher than ex situ HAP/PAAc composite.

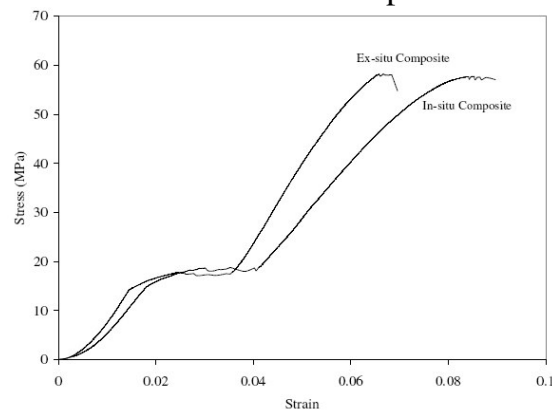

Fig. 3: Plot Showing Stress-Strain curve of compression test on dry 70/30(HAP/Polymer) samples.

In situ HAP contains $30 \%$ PAAc by weight, thus in in situ HAP/PAAc composite total weight $\%$ of PAAc is around $51 \%$. On the other hand, ex situ HAP/PAAc contains $30 \mathrm{wt}$. $\%$ of PAAc. It's interesting to note 
comparable elastic modulus of in situ and ex situ HAP/PAAc composite in spite of lower mineral content in in situ HAP/PAAc composite. The improved mechanical response of in situ HAP/PAAc composite can be attributed to stronger interfacial interaction between HAP crystals and PAAc. The onset of plastic deformation in first stage is at 0.014 and 0.016 strain for in situ and ex situ composites respectively. Compressive strength of both ex situ and in situ composite is similar and is around $58 \mathrm{MPa}$, However, in situ composite samples exhibit higher strain at failure (0.09 strain). Figure 4 shows stress/strain plots of SBF soaked samples. Both in situ and ex situ composites show large strain after soaking in SBF for $2 \mathrm{~min}$.

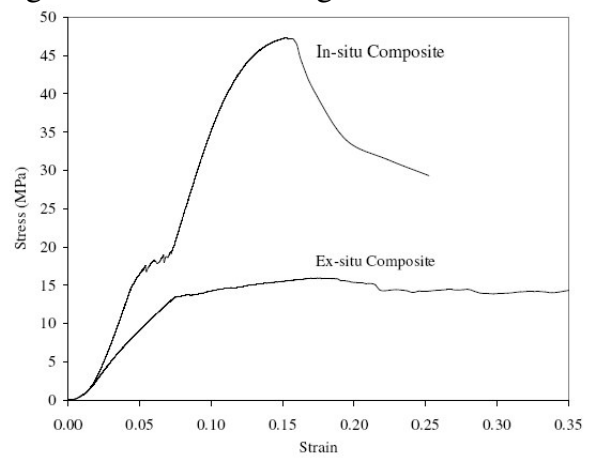

Fig. 4: Plot Showing Stress-Strain curve of compression test on 70/30 (HAP/Polymer) sample soaked in SBF.

The onset of plastic deformation of in situ and ex situ composites in first stage is around 0.05 and 0.08 strain respectively. Although, elastic modulus of in situ composites decreased significantly, but it was found to be higher than SBF soaked ex situ composites. These results suggest that SBF acts as a plasticizer. SBF soaked in situ composites show much lower compressive strength (47 $\mathrm{MPa})$ than dry in situ samples. SBF soaked ex situ composite exhibit large deformation before they fail at 0.40 strain. Compressive strength of SBF soaked ex situ composites was significantly lower than ex situ dry composites. Figure 5 shows stress/strain plots of in situ and ex situ composites soaked in water for $2 \mathrm{~min}$.

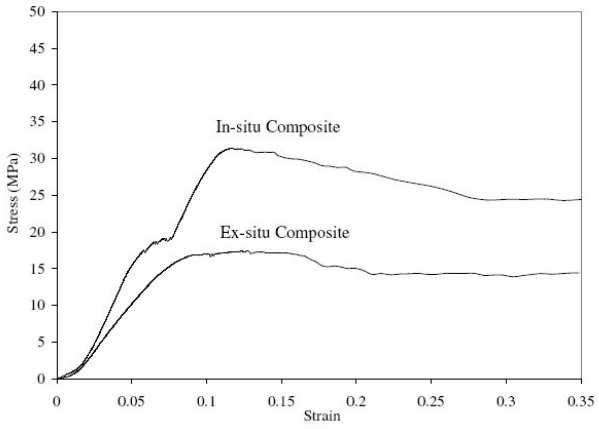

Fig. 5: Plot Showing Stress-Strain curve of compression test on 70/30(HAP/Polymer) sample soaked in deionized water.

Water soaked ex situ composites showed similar response as SBF soaked ex situ composites. Water soaked in situ composites showed lower compressive strength than SBF soaked in situ composites. The effect of water soaking is more deteriorating on mechanical properties than SBF. Figure 6 and 7 show stress/strain plots of 60/40 HAP/PAAc composites soaked in SBF and water respectively. These samples are also soaked for $2 \mathrm{~min}$. either in water or SBF before mechanical tests were performed. Unlike 70/30 ex situ HAP/PAAc, water and SBF soaked composites, 60/40 ex situ HAP/PAAc water and SBF soaked composites show two a stage behavior. In in situ composite, not only do the $\mathrm{Ca}$ atoms but also carboxylate groups of in situ HAP interact with carboxylate of PAAc polymer (Figure 8).

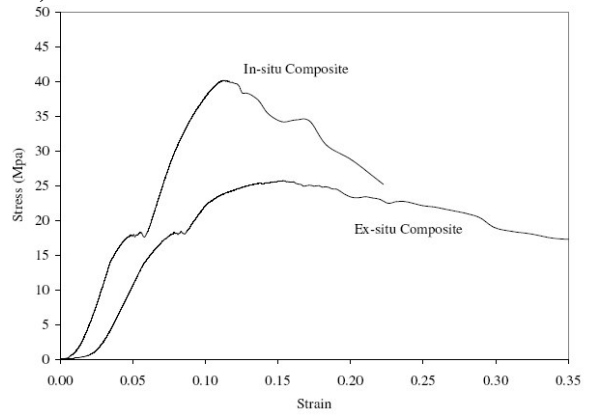

Fig. 6: Plot Showing Stress-Strain curve of compression test on 60/40(HAP/Polymer) samples soaked in SBF.

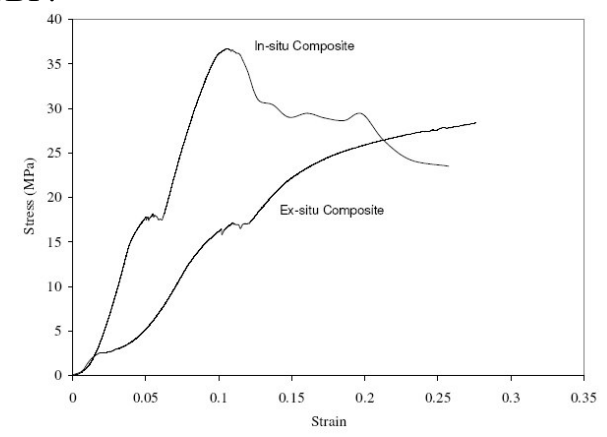

Fig. 7: Plot Showing Stress-Strain curve of compression test on 60/40 (HAP/Polymer) samples soaked in de-ionized water

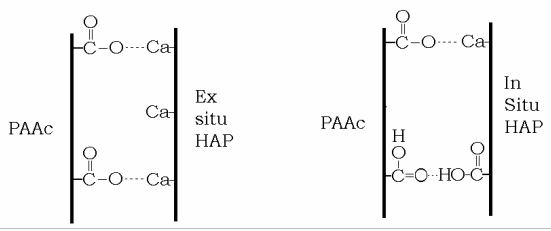

Fig. 8: Schematic showing interfacial interactions in ex situ and in situ composites

On the other hand, in ex situ composites, only $\mathrm{Ca}$ atoms of ex situ HAP interact with carboxylate groups of PAAc resulting in a weaker interface as compared to interface in situ composites. Since, these composites are prepared by compacting at room temperature; the interface between HAP-HAP particles is further weakened. When, ex situ composites are soaked in water or SBS, the adsorbed liquid phase causes further weakening of interface between HAP and polymer. 
This is the reason why SBF and water soaked ex situ ex situ composites are not able to withstand higher load and collapse. The mechanical response of SBF and water soaked 60/40 composites is better than 70/30 composites samples. This is due to lesser HAP-HAP interface in $60 / 40$ composites compared to $70 / 30$ composites.

Figure 9 shows loading and unloading curve of ex situ and in situ HAP/PAAc dry composites. The loading and unloading curve of composites soaked in water is shown in Figure 10. Figure 11 shows loading/unloading curve of water soaked composites. Both ex situ and in situ HAP/PAAc samples show large residual strain and creep like behavior after soaking in SBF and water. The effect of strain rate on composites was also studied. Tests were performed on dry samples and different strain rate applied are $0.001,0.01,0.1$ and $1 \mathrm{sec}-1$. Effect of strain rate on elastic modulus of ex situ and in situ composites are shown in Figure 12. Highest elastic modulus was observed at $0.01 \mathrm{sec}-1$ for both ex situ and in situ composites. Strain at failure is shown in Figure 13.
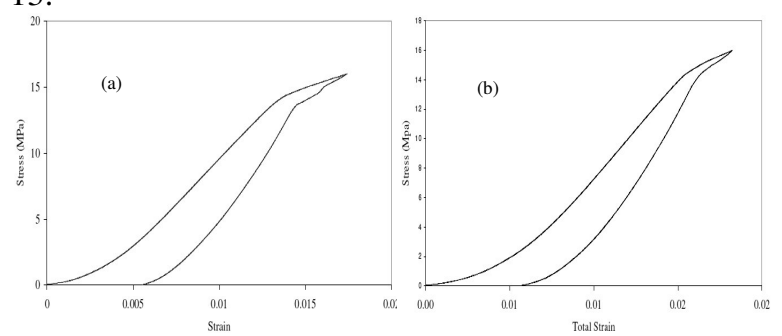

Fig. 9: Plot Showing Stress-Strain curve of unloading test on dry 70/30 (HAP/Polymer) (a) ex situ sample. (b) in situ sample

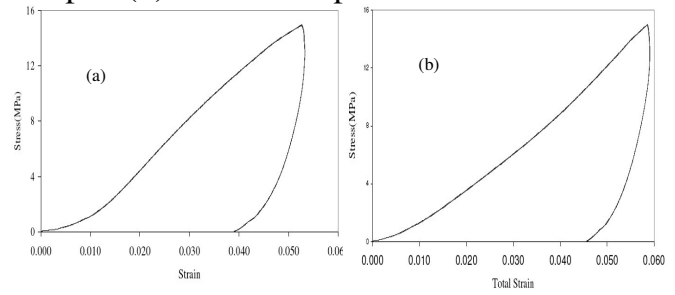

Fig. 10: Plot Showing Stress-Strain curve of unloading test on 70/30 (HAP/Polymer) soaked in de-ionized water for 2 min. (a) ex situ sample (b) in situ
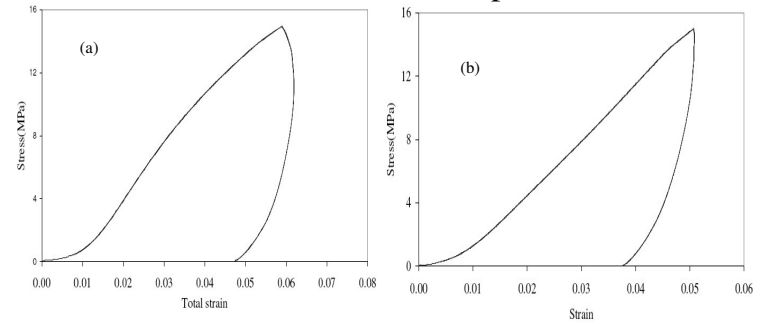

Fig. 11: Plot Showing Stress-Strain curve of unloading test on 70/30 (HAP/Polymer) soaked in simulated body fluid for 2 min. (a) ex situ composite (b) in situ composite.

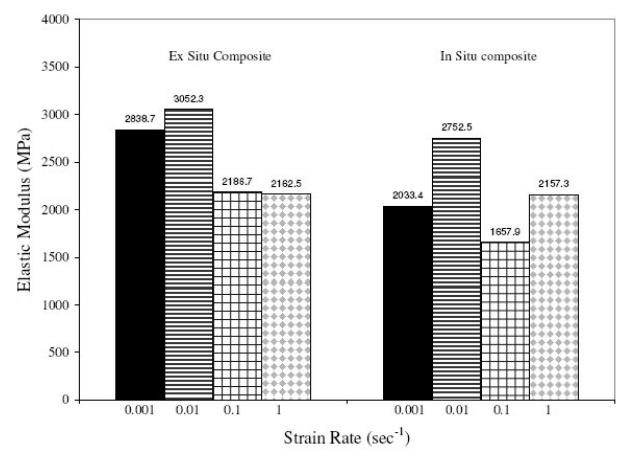

Fig. 12: Chart shows elastic modulus of 70/30 ex situ and in situ composites in compression test with a strain rate of $0.001 / \mathrm{sec}, 0.01 / \mathrm{sec}, 0.1 / \mathrm{sec}$ and $1 / \mathrm{sec}$.

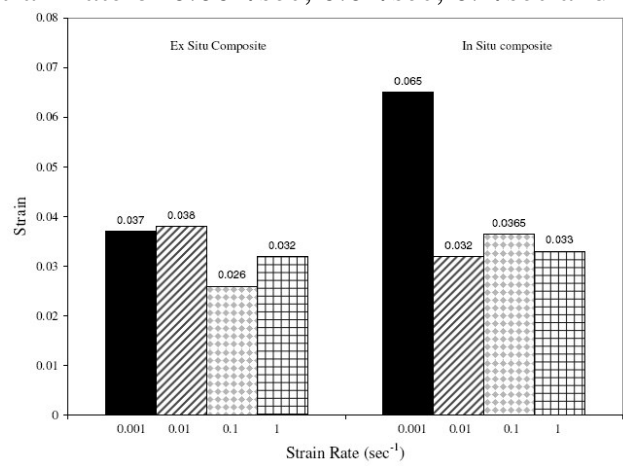

Fig. 13: Chart shows strain at failure of 70/30 ex situ and in situ composites in compression test with a strain rate of $0.001 / \mathrm{sec}, 0.01 / \mathrm{sec}, 0.1 / \mathrm{sec}$ and $1 / \mathrm{sec}$.

It is observed that in situ composite show higher strain to failure at 0.001 sec-1 compared to ex situ composites. However, at $1 \mathrm{sec}-1$ strain rate both composites show comparable strain to failure. Figure 14 shows variation in compressive strength of both composites at different strain rate. Ex situ composites show the highest compressive strength of $81 \mathrm{MPa}$ at $0.01 \mathrm{sec}-1$ and lowest at 0.1 and $1 \mathrm{sec}-1$ strain rate. Furthermore in, in situ composites compressive strength decreases as strain rate is decreased. These composites show highest compressive strength of $108 \mathrm{MPa}$ at strain rate of $0.001 \mathrm{sec}^{-1}$ and lowest compressive strength of $32 \mathrm{MPa}$ at strain rate of $1 \mathrm{sec}^{-1}$.

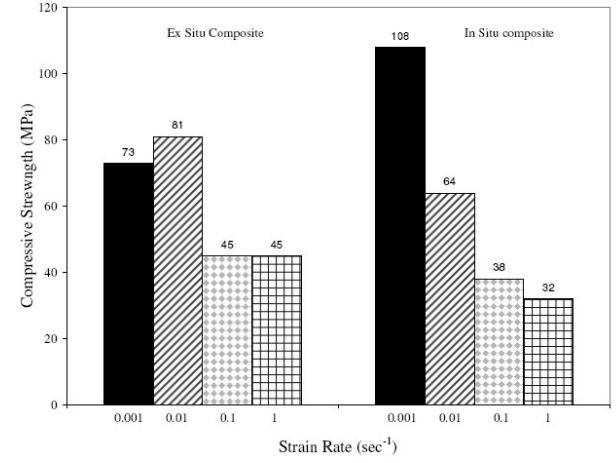

Fig. 14: Chart shows compressive strength of 70/30 ex situ and in situ composites in compression test with a strain rate of $0.001 / \mathrm{sec}, 0.01 / \mathrm{sec}, 0.1 / \mathrm{sec}$ and $1 / \mathrm{sec}$. 
$\mathrm{HAP}$ /polymer composites prepared at 70/30 proportions and soaked in simulated body fluid (SBF) and deionized water for 3 hours, are analyzed using XRD. Two calcium phosphate $\left(\mathrm{Ca}_{2} \mathrm{P}_{2} \mathrm{O}_{7}\right)$ phases and a hydroxyapatite phase are observed for ex situ SBF and water soaked composites using XRD (Figure 15).

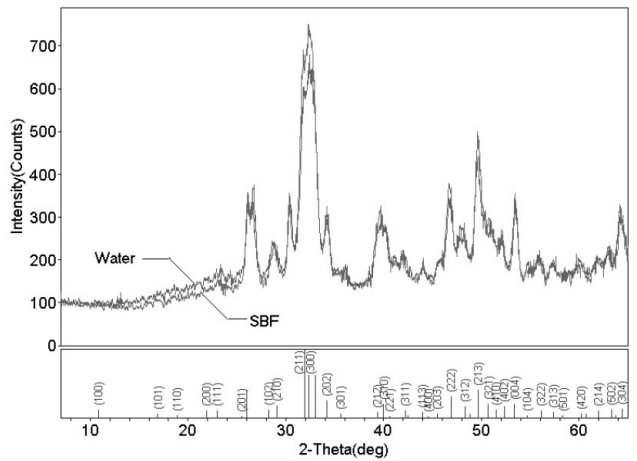

Fig. 15: XRD plot of ex situ 70/30 (HAP/polymer) samples soaked in water and SBF for 3 hours.

In addition to these phases, other salts including a synthetic halite-like phase may also be present in trace quantities. Figure 16 shows the XRD plots of in situ 70/30 composite soaked in SBF for 3hrs. This figure indicates the presence of hydroxyapatite phase only. The in situ 70/30 composites are appears to have a lower crystallinity when compared to ex situ composites.

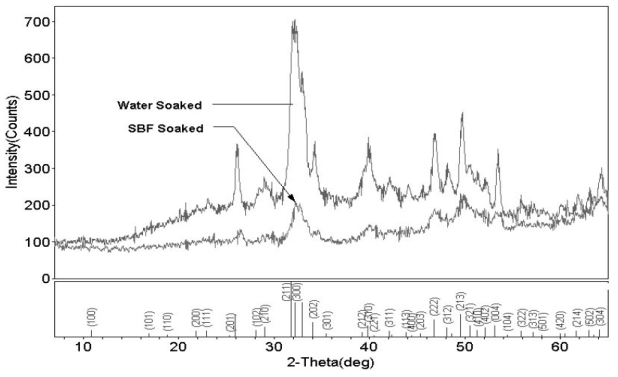

Fig. 16: XRD plot of in situ 70/30 (HAP/polymer) samples soaked in water and SBF for 3 hours.

\section{CONCLUSIONS}

Composites of insitu mineralized hydroxyapatite with PAAc have been investigated. Interfacial interactions play an important role on mechanical behavior in SBF and water soaked samples. The mechanical response of the 70/30 and 60/40 composites under wet conditions suggests that SBF and water weakens HAP-HAP interface significantly. PAS-FTIR suggests that PAAc attach to HAP by dissociated carboxylate groups. The water and SBF soaked samples showed creep like behavior and contained large residual strain after unloading. Loading under different strain rates has significant effect on mechanical properties of these composites. Both in situ and ex situ 70:30 composites exhibit highest elastic modulus at $0.01 \mathrm{sec}^{-1}$ strain rate. XRD study indicates formation of $\mathrm{Ca}_{2} \mathrm{P}_{2} \mathrm{O}_{7}$ phase in ex situ composite after soaking in $\mathrm{SBF}$ and water for 3 hours, whereas in situ composites showed presence of only hydroxyapatite phase after soaking in SBF and water for same duration of time.

\section{ACKNOWLEDGMENTS}

This work was supported by grant from National Science Foundation (CAREER \# 0132768).

\section{REFERENCES}

1. Mayer, G., 2005. Rigid biological systems as models for synthetic composites. Science, 310: 1144-1147.

2. Zhang, S., 2003. Fabrication of novel biomaterials through molecular self-assembly. Nature Biotechnology, 21: 1171-1178.

3. Lutolf, M.P. and J.A. Hubbell, 2005. Synthetic biomaterials as instructive extracellular microenvironments for morphogenesis in tissue engineering. Nature Biotechnology, 23: 47-55.

4. Katti, K.S., D.R. Katti, S.M. Pradhan and A. Bhosle, 2005. Platelet interlocks are the key to toughness and strength in nacre, Journal of Materials Research, 20: 1097-1100.

5. Nudelman, F., B.A. Gotliv, L. Addadi and S. Weiner, 2006. Mollusk shell formation: Mapping the distribution of organic matrix components underlying a single aragonitic tablet in nacre. Journal of Structural Biology, 153:176-187.

6. Gotliv, B.A., L. Addadi and S. Weiner, 2003. Mollusk shell acidic proteins: In search of individual functions. ChemBioChem, 4: 522-529.

7. Dalas, E., J.K. Kallitsis and P.G. Koutsoukos, 1991. Crystallization of hydroxyapatite on polymers. Langmuir, 7:1822-1826.

8. Skrtic, D. and E.D. Eanes, 1993. Effect of membrane cholesterol on calcium phosphate formation in aqueous suspensions of anionic liposomes. Calcified Tissue Internat., 50:55-60.

9. Zhang ,L.J., H.G. Liu, X.S. Feng, DJ. Qian, L. Zhang, X.L. Yu and Q.L. Feng, 2004. Study on growth of hydroxyapatite induced by dipalmitoylphosphatidylcholine Langmuir monolayer. Thin Solid Films, 458:287-291.

10. Gupta, Y., Mathur, G.N., Verma, S. Biomimetic synthesis and ultrastructural characterization of a zerovalent gold-hydroxyapatite composite ,(2006) Bioorganic and Medicinal Chemistry Letters, 16 2, Pages 363-366.

11. Podsiadlo, P., Paternel, S., Rouillard, J.-M., Zhang, Z., Lee, J., Lee, J.-W., Gulari, E., Kotov, N.A. Layer-by-layer assembly of nacre-like nanostructured composites with antimicrobial properties ,(2005) Langmuir, 21 25, Pages 11915-11921.

12. Brown, E.N. Biomimetic composites: Inspiration to application, (2005) Proceedings of the 2005 SEM Annual Conference and Exposition on Experimental and Applied Mechanics, Pages 245249. 
13. Zhang, X.-A., Wu, W.-J., Liu, C.-L., Wang, J.-F. Self-assembled organic/inorganic layered composite materials ,(2005) Gongneng Cailiao/Journal of Functional Materials, 36 11, Pages 1645-1650.

14. Belcher, A.M., P.K. Hansama, G.D. Stucky and D.E. Morse, 1998. First steps in harnessing the potential of biomineralization as a route to new high-performance composite materials. Acta Mater, 46: 733-736.

15. Mann, S., D.D. Archibald, J.M. Didymus, T. Douglas, B.R. Heywood, F.C. Meldrum and N.J. Reeves, 1993. Crystallization at inorganic-organic interfaces: Biomaterials and biomimetic synthesis. Science, 261:1286-1292.

16. Kato, K., Y. Eika and Y. Ikada, 1997. In situ hydroxyapatite crystallization for the formation of hydroxyapatite/polymer composites. Journal of Materials Science, 32:5533-5543.

17. Koutsopoulos, S., J. Demakopoulos, X. Argiriou, E. Dalas,N. Klouras and N. Spanos. 1995. Inhibition of Hydroxyapatite Formation by Zirconocenes. Langmuir, 11: 1831-1834.

18. Bonfield, W., 1998. Annals of the New York Academy of Sciences, 523, edited by P. Ducheyne and L. E. Lemons, NewYork, pp. 173.

19. Chang, M.C., C.C. Ko and Douglas W.H., 2003. Preparation of hydroxyapatite-gelatin nanocomposite. Biomaterials, 24:2853-2862.

20. Katti, K. and P. Gujjula, 2002. Control of mechanical responses in in situ polymerhydroxyapatite composites for bone replacement. Proc. 15th ASCE Engineering Mechanics Conf. New York, NY.

21. Katti, K. and P. Gujjula, 2002. In situ mineralization of hydroxyapatite for a molecular control of mechanical responses in hydroxyapatitepolymer composites for bone replacement. Materials Research Society Proceeding, Boston, MA, 711: GG4.3.1.-GG4.3.6.

22. Verma, D., K. Katti and D. Katti, 2006. Experimental investigation of interfaces in hydroxyapatite/polyacrylic acid/polycaprolactone composites using photoacoustic FTIR spectroscopy. J Biomed Mater Res., 77A: 59-66.

23. Verma, D., K. Katti and D. Katti, 2006. Bioactivity in in situ hydroxyapatite-polycaprolactone composites. Journal of Biomedical Materials Research, in press.

24. Bhowmik, R., K. S. Katti, D. Verma D. R. Katti, 2006. Probing Molecular Interactions in Bone Biomaterials: Through Molecular Dynamics and Fourier Transform Infrared Spectroscopy, to appear in Mater. Sci. Eng. C.

25. Dauber-Osguthorpe, P., V.A. Roberts, D.J. Osguthorpe, J. Wolff, M. Genest and A.T. Hagler, 1988. Structure and energetics of ligand binding to proteins: escherichia coli dihydrofolate reductasetrimethoprim, a drug-receptor system. Proteins: Structure, Function and Genetics, 4:31-47.
26. Maple, J., U. Dinur, and A.T. Hagler, 1988. Derivation of Force Fields for Molecular Mechanics and Dynamics from ab initio Energy Surfaces. Proc. Nat. Acad. Sci. U.S.A., 85:53505354.

27. Ritschla, F., M. Faitb, K. Fiedlera, J.E.H. Köhlerc, B. Kubiasb and M. Meisela, 2002. An extension of the consistent valence force field (CVFF) with the aim to simulate the structures of vanadium phosphorous oxides and the adsorption of $n$-butane on their crystal planes. Z. Anorg. Allg. Chem., 628:1385-1396.

28. Katti, K. S., D. Sikdar, D. R. Katti, P. Ghosh, D. Verma, 2006. Molecular Interactions In Intercalated Organically Modified Clay In ClayPolycaprolactam Nanocomposites: Experiments And Modeling, Polymer 47, 403-414

29. Schmidt, S., D.R. Katti, P.Ghosh, and K.S. Katti, 2005. Evolution of Mechanical Response of Sodium Montmorillonite Interlayer with Increasing Hydration, Langmuir, 21, 8069-8076.

30. Katti, D.R., S. Schmidt, P. Ghosh, and K.S.Katti, 2005. Modeling Response of Pyrophyllite Clay Interlayer to Applied Stress Using Steered Molecular Dynamics, Clays and Clay Minerals, 52, 171-178.

31. D.R. Katti, Ghosh, P., Schmidt, S. and Katti, K.S., 2005. Mechanical properties of sodium montmorillonite interlayer intercalated with amino acids, Biomacromolecules 6, 3276-3282.

32. Katti, K.S., R. Bhowmik, and D.R. Katti, Molecular modeling of hydroxyapatite-

polyacrylic acid interface. Under review, Biomacromolecules

33. Gardebien, F., Jean-Luc Bredas and R. Lazzaroni, 2005. Molecular dynamics simulations of nanocomposites based on poly ( $\varepsilon$-caprolactone) grafted on montmorillonite clay. J. Phys. Chem. B., 109:12287-12296.

34. Fermeglia, M., M. Ferrone and S. Pricl, 2004. Estimation of the Binding energy in random poly (butylenes terephtalate-co-thiodiethylene terephtalate) copolyesters/clay nanocomposites via molecular simulations. Molecular Simulation, 30:289-300.

35. Shevade, A. V., M.A. Ryan, M.L. Homer, A.M. Manfreda, H. Zhou and K.S. Manatt, 2003. Molecular modeling of polymer composite-analyte interactions in electronic nose sensors. Sensors and Actuators B, 93:84-91.

36. Fermeglia, M., M. Ferrone, and S. Pricl, 2003. Computer simulation of nylon-6/organoclay nanocomposites: prediction of binding energy. Fluid Phase Equilibria, 212:315-329. 